Tropical Journal of Pharmaceutical Research February 2019; 18 (2): 371-375

ISSN: $1596-5996$ (print); 1596-9827 (electronic)

(C) Pharmacotherapy Group, Faculty of Pharmacy, University of Benin, Benin City, 300001 Nigeria.

\title{
Therapeutic effect of co-administered salvianolate and atorvastatin calcium on coronary heart disease patients with angina pectoris, and their blood lipid levels
}

\author{
Guangyu Cheng ${ }^{1}$, Qingjie $\mathrm{Li}^{1}$, Lin $\mathrm{Li}^{1}$, Qi Wang ${ }^{2}$, Aidong $\mathrm{Liu}^{3 *}$ \\ ${ }^{1}$ Research Center of Traditional Chinese Medicine, Affiliated Hospital to Changchun University of Chinese Medicine, \\ Changchun, Jilin 130021, ${ }^{2}$ College of Chinese Material Medicine, Jilin Agricultural University, Jilin 130117, ${ }^{3}$ The Department of \\ Cardiology, Affiliated Hospital to Changchun University of Chinese Medicine, Changchun, Jilin 130021, China
}

*For correspondence: Email: ps1167@163.com

Sent for review: 24 October 2018

Revised accepted: 21 January 2019

\begin{abstract}
Purpose: To investigate the clinical effect of combination of salvianolate and atorvastatin on blood lipids of coronary heart disease patients with angina pectoris (CHD-AP).

Method: Patients with CHD-AP $(n=104)$ from January 2016 to January 2017 were randomly assigned to two groups: control group treated with atorvastatin (10 mg/day), and study group was administered atorvastatin (10 mg/day, oral) plus salvianolate $(200 \mathrm{mg} /$ day in $5 \%$ glucose, iv). Palpitation, chest distress, improvement in myocardial ischemia, myocardial function, and hemodynamics were determined and used to assess treatment effectiveness in the two groups. Differences in blood lipid profiles were also investigated.

Results: Improvement in palpitation, chest distress, myocardial ischemia and myocardial function in the study group were significantly higher than in the control group $(p<0.05)$. In the study group, total cholesterol (TC), low density lipoprotein cholesterol (LDL-C) and triacylglycerols (TGs) significantly decreased, relative to the control group $(p<0.05)$.

Conclusion: Treatment of CHD-AP patients with combination of salvianolate and atorvastatin significantly ameliorates coronary heart disease and angina pectoris, and also reduces their blood lipid levels.
\end{abstract}

Keywords: Salvianolate, Atorvastatin, Coronary heart disease, Angina pectoris, Blood lipids

\begin{abstract}
This is an Open Access article that uses a funding model which does not charge readers or their institutions for access and distributed under the terms of the Creative Commons Attribution License (http://creativecommons.org/licenses/by/4.0) and the Budapest Open Access Initiative (http://www.budapestopenaccessinitiative.org/read), which permit unrestricted use, distribution, and reproduction in any medium, provided the original work is properly credited.

Tropical Journal of Pharmaceutical Research is indexed by Science Citation Index (SciSearch), Scopus, International Pharmaceutical Abstract, Chemical Abstracts, Embase, Index Copernicus, EBSCO, African Index Medicus, JournalSeek, Journal Citation Reports/Science Edition, Directory of Open Access Journals (DOAJ), African Journal Online, Bioline International, Open-J-Gate and Pharmacy Abstracts
\end{abstract}

\section{INTRODUCTION}

Coronary heart disease (CHD) is a clinically common cardiovascular disease with high mortality and disability. The safety of life of patients with $\mathrm{CHD}$ is seriously threatened. The disease results from changes in coronary function. Coronary atherosclerosis causes transient myocardial ischemia or hypoxia, leading to chest pain which is the main clinical manifestation of the syndrome [1]. Coronary heart disease (CHD) is often associated with hyperlipidemia due to significant elevation of blood TC and TGs, and decreased blood levels of HDL-C. Total cholesterol (TC) and TG levels are significantly elevated, often accompanied by 
decreases in HDL-C. Decreases in HDL-C and elevated LDL-C are important risk factors for the occurrence of $\mathrm{CHD}$ [2].

Salvianolate is a medicinally active component of Salvia miltiorrhizae, a traditional and natural medicine for enhancing blood flow and eliminating blood stasis. It is popularly employed for treating CHD and AP. Salvia miltiorrhizae contains lipid-soluble and water-soluble phenolic compounds, with salvianolate as the most important water-soluble active component. Salvianolate is employed as a therapeutic agent for diseases associated with the cardiovascular system [3]. Statins are clinically used as intensive lipid-lowering drugs. They act through a mechanism involving specific inhibition of HMGCoA reductase activity, thereby inhibiting cholesterol biosynthesis, and reducing the blood levels of TC and LDL-C [4]. The present study was carried out to assess the impact of combined administration of salvianolate injection and atorvastatin calcium on patients with CHDAP, and its effect on blood lipid profiles.

\section{METHODS}

\section{Subjects}

A total of 104 patients with CHD-AP from January 2016 to January 2017 were enrolled in our hospital. They comprised 50 men and 54 women within the age range of $45-75$ years (mean age $=58$ years), with weekly incidence of angina pectoris $\geq 2$. Patients with drug allergy, pregnant and lactating patients, patients with severe damage to the major organs (heart, liver and kidney); cancer patients, patients who underwent interventional therapy and coronary artery bypass grafting, and patients with mental disorders were excluded. The diagnostic and inclusion criteria used in this clinical study were in line with the diagnostic criteria for CHD angina in the Nomenclature and Diagnostic Criteria for Ischemic Heart Disease. The study received approval from the ethics committee of Affiliated Hospital to Changchun University of Chinese Medicine (Approval No. CUCM-2017043), and was performed in line with the Helsinki declaration of 1964 as amended in 1996 [5].

\section{Treatments}

The patients $(\mathrm{n}=104)$ were randomly and double-blindly divided into study and control groups (52 patients per group). As shown in Table 1, both groups were comparable with respect to age, gender, and average number of angina punctures.
Table 1: Basic profile of patients

\begin{tabular}{lccc}
\hline Group & Male & $\begin{array}{c}\text { Mean age } \\
\text { (years) }\end{array}$ & $\begin{array}{c}\text { Number of angina } \\
\text { punctures } \\
\text { per week }\end{array}$ \\
\hline Study & 24 & $\begin{array}{c}58.91 \pm \\
3.94\end{array}$ & $2.31 \pm 0.22$ \\
Control & 26 & $\begin{array}{c}57.05 \pm \\
4.18\end{array}$ \\
$x^{2}$ & 0.353 & 0.865 & $2.55 \pm 0.34$ \\
$p$ & 0.527 & 0.278 & 0.154 \\
\hline
\end{tabular}

Patients in both groups were given routine treatment. In addition to the conventional treatment, the control group received oral atorvastatin calcium tablets (Pfizer Ireland Pharmaceuticals Production, approval no.: Chinese medicine standard 15J2200) at a dose of $10 \mathrm{mg} / \mathrm{day}, 30 \mathrm{~min}$ after dinner. The study group was given routine treatment and oral atorvastatin calcium tablets (10 mg/day), in addition to intravenous infusion of salvianolate (Shanghai Green Valley Pharmaceutical Co. Ltd., Guoyao Zhunzizhong 0050249) at a dose of 200 $\mathrm{mg} / \mathrm{day}$ in $5 \%$ glucose. Treatment in the two groups lasted for 8 weeks [6].

\section{Evaluation of clinical effectiveness}

Clinical effectiveness was evaluated as outlined earlier [7]. The categorizations used were as follows:

Effective: Implied disappearance of palpitations and chest tightness; normalization of patient's ECG or dynamic ECG ST segment or T wave; significant effect: Implied disappearance of palpitations and chest tightness; improvement in ECG or dynamic electrocardiogram ST-T without normalization, e.g., ST segment rebound $>0.05$ $\mathrm{mV}$, low $\mathrm{T}$ wave, or $\mathrm{T}$ wave recovery from low to erect, or decrease of ST segment to $0.05 \mathrm{mV}$ $0.10 \mathrm{mV}$; and invalid: No improvement in palpitation, chest tightness and ECG ST-T.

\section{Determination of cardiac function and hemodynamics}

SA9000 Hemodynamic Tester was used to measure cardiac function. The parameters measured were ejection fraction (EF), cardiac output (CO), as well as hemodynamics such as plasma viscosity (PV).), whole blood viscosity high cut (WBVHC) and platelet adhesion rate (PAR) [8].

\section{Evaluation of blood lipid levels}

The blood lipid profiles of the study group and the control group before and after treatment were measured. Fasting venous blood $(3 \mathrm{~mL})$ was 
taken from each patient in the morning, and the serum was separated by centrifugation within 2 h. The TC levels were determined by the endpoint method, and glycerol was determined by the oxidase method. The serum levels of TGs, HDL-C and LDL-C were determined using the procedures described in a previous study [9].

\section{Statistical analysis}

Count data are presented as percentage, while measurement data are presented as mean \pm standard deviation (SD). Groups were compared using $t$-test. All statistical analyses were done with SPSS version 19.0 software. Values of $p<$ 0.05 were assumed significant.

\section{RESULTS}

\section{Amelioration of palpitation and chest tightness}

After 8 weeks of treatment, improvements in palpitations and chest tightness were observed. Table 1 shows that the total effectiveness in the control and study groups were $71.2 \%$ and 92.3 $\%$, respectively. Improvements in palpitations and chest tightness were significantly higher in the study group than in the control group ( $p<$ 0.05).

\section{Amelioration of myocardial ischemia}

Table 3 shows that the total effectiveness in the control and study groups were 61.5 and $82.7 \%$, respectively, with greater amelioration of myocardial ischemia in study group patients than in control patients $(p<0.05)$.

\section{Cardiac function}

Before treatment, cardiac function parameters (EF and CO) were comparable in the two groups. However, there were significant post-treatment increases in EF and $\mathrm{CO}$ in both groups, with higher increase in the study group $(p<0.05)$, as shown in Table 4.

\section{Hemodynamic parameters}

Pre-treatment hemodynamic function parameters (PV, WBVHC and PAR) were comparable between the two groups (Table 5). However, after treatment, these parameters significantly decreased in the study group $(p<0.05)$.

Table 2: Effect of treatment on palpitations and chest tightness

\begin{tabular}{lcccc}
\hline Group & $\begin{array}{c}\text { Significantly } \\
\text { effective }\end{array}$ & Effective & Ineffective & $\begin{array}{c}\text { Overall effectiveness } \\
(\%)\end{array}$ \\
\hline Control & 21 & 16 & 15 & 71.2 \\
Study & 31 & 17 & 4 & $92.3^{*}$ \\
\hline${ }^{*} P<0.05$, compared with the control group & & &
\end{tabular}

Table 3: Effect of treatment on myocardial ischemia

\begin{tabular}{lcccc}
\hline Group & Effective & $\begin{array}{c}\text { Significantly } \\
\text { effective }\end{array}$ & Ineffective & $\begin{array}{c}\text { Overall effectiveness } \\
(\%)\end{array}$ \\
\hline Control & 19 & 13 & 20 & 61.5 \\
Study & 28 & 15 & 9 & $82.7^{*}$ \\
\hline${ }^{*} P<0.05$, relative to control group & & &
\end{tabular}

Table 4: Effect of treatment on cardiac function (mean \pm SD)

\begin{tabular}{|c|c|c|c|c|}
\hline \multirow{2}{*}{$\begin{array}{l}\text { Cardiac function } \\
\text { index }\end{array}$} & \multicolumn{2}{|c|}{ Control group } & \multicolumn{2}{|c|}{ Study group } \\
\hline & Before treatment & After treatment & $\begin{array}{l}\text { Before } \\
\text { treatment }\end{array}$ & After treatment \\
\hline EF (\%) & $43.22 \pm 3.55$ & $54.21 \pm 2.55^{*}$ & $42.98 \pm 3.12$ & $69.22 \pm 3.66^{\star} \#$ \\
\hline $\mathrm{CO}(\mathrm{L} / \mathrm{min})$ & $42.54 \pm 3.21$ & $50.33 \pm 3.14^{*}$ & $43.67 \pm 3.45$ & $58.34 \pm 2.88^{*} \#$ \\
\hline
\end{tabular}

${ }^{\star \star} P<0.05$, relative to pre-treatment value; $\# p<0.05$, control group relative to study group

Table 5: Impact of treatments on hemodynamic parameters (mean \pm SD)

\begin{tabular}{lccccc}
\hline \multirow{2}{*}{ Hemodynamic index } & \multicolumn{2}{c}{ Control group } & & \multicolumn{2}{c}{ Study group } \\
\cline { 2 - 3 } \cline { 5 - 6 } & Before treatment & After treatment & & Before treatment & After treatment \\
\hline PV $(\mathrm{mPa} \cdot \mathrm{s})$ & $3.66 \pm 0.55$ & $3.02 \pm 0.32^{*}$ & & $3.71 \pm 0.76$ & $2.32 \pm 0.23^{\star} \#$ \\
WBVHC $(\mathrm{mPa} \cdot \mathrm{s})$ & $2.43 \pm 0.34$ & $1.98 \pm 0.33^{*}$ & & $2.49 \pm 0.35$ & $1.66 \pm 0.23^{*} \#$ \\
PAR $(\%)$ & $6.55 \pm 1.21$ & $5.12 \pm 1.14^{*}$ & & $6.33 \pm 1.01$ & $4.22 \pm 0.88^{*} \#$ \\
\hline${ }^{*} p<0.05$, relative to pre-treatment value; $\# p<0.05$ & & &
\end{tabular}


Table 6: Effect of treatment on blood lipid levels (mean \pm SD)

\begin{tabular}{lccccc}
\hline \multirow{2}{*}{ Blood lipid } & \multicolumn{2}{c}{ Control group } & & \multicolumn{2}{c}{ Study group } \\
\cline { 2 - 3 } \cline { 5 - 6 } & Before treatment & After treatment & & Before treatment & After treatment \\
\hline TC $(\mathrm{mM})$ & $6.56 \pm 1.44$ & $5.27 \pm 0.81^{*}$ & & $6.67 \pm 1.56$ & $4.26 \pm 0.77^{\star} \#$ \\
TG $(\mathrm{mM})$ & $2.45 \pm 0.41$ & $2.03 \pm 0.39^{*}$ & & $2.43 \pm 0.44$ & $1.66 \pm 0.51^{\star} \#$ \\
HDL-C $(\mathrm{mM})$ & $1.31 \pm 0.40$ & $1.41 \pm 0.31$ & & $1.32 \pm 0.51$ & $1.61 \pm 0.45$ \\
LDL-C $(\mathrm{mM})$ & $3.70 \pm 0.81$ & $2.98 \pm 0.77^{*}$ & & $3.75 \pm 0.85$ & $2.25 \pm 0.66^{*} \#$ \\
\hline${ }^{*} P<0.05$, relative to pre-treatment; $\# p<0.05$, study group vs. control group
\end{tabular}

${ }^{\star} P<0.05$, relative to pre-treatment; $\# p<0.05$, study group vs. control group

Effect of combination therapy on blood lipid profiles

As depicted in Table 6, before treatment, in blood lipid levels did not differ significantly between the two groups $(p>0.05)$. However, 8 weeks posttreatment, the blood lipid levels of both groups were significantly improved, with better improvements in the study group patients than in control patients $(p<0.05)$.

\section{DISCUSSION}

Coronary heart disease is an often-encountered cardiovascular condition in clinics. It is one of the common causes of death in patients. The pathological mechanism involved in the disease is vascular atherosclerosis. At present, CHD-AP occurs at younger ages, unlike before. In severe cases, CHD-AP degenerates into myocardial infarction leading to sudden death $[9,10]$. The main strategy for treating CHD-AP involves increasing the tolerance to myocardial ischemia/hypoxia, thereby enhancing myocardial microcirculation and improving myocardial blood supply.

Elevated blood lipid levels are also important factors in angina pectoris. High blood lipids are associated with marked elevations in blood TC and triglycerides, often accompanied with lowered HDL-C and elevated LDL-C. Raised blood levels of TC, TGs LDL-C are important risk elements for coronary heart disease. Atorvastatin calcium effectively inhibits endogenous synthesis of cholesterol and effectively regulates blood lipid levels by reducing blood levels of TC and LDL-C. It does these through a mechanism involving specific inhibition of HMG-CoA reductase, thereby reducing the incidence of atherosclerosis-induced angina pectoris [11].

Salvia miltiorrhizae is used as a drug to improve blood circulation, remove blood stasis and nourish blood, and it produces remarkable curative effects without adverse reactions [12]. Salvia polyphenolate, a water-soluble active compound isolated from Salvia miltiorrhizae, is widely used in the treatment of cardiovascular diseases. Its mechanism of action includes protection of myocardial membrane from damage, and inhibition of atherosclerosis [13, 14].

These results demonstrate that combination of Danshen polyphenolate and atorvastatin calcium tablets in the treatment of CHD-AP produces significantly better effectiveness than the use of atorvastatin calcium tablets alone.

\section{Study limitations}

Due to limitations arising from short treatment duration and small sample size, the results obtained in this study may have certain errors. Thus, it is necessary to expand the number of subjects, and increase patients' disease tracking time in further studies.

\section{CONCLUSION}

The results obtained this study indicate that combined treatment of salvianolate and atorvastatin calcium tablets results in significantly improved therapeutic outcomes in CHD-AP patients, as well as significant reductions in their blood lipid profiles.

\section{DECLARATIONS}

\section{Conflict of Interest}

No conflict of interest associated with this work.

\section{Contribution of Authors}

We declare that this work was done by the author(s) named in this article and all liabilities pertaining to claims relating to the content of this article will be borne by the authors. All authors read and approved the manuscript for publication. Aidong Liu conceived and designed the study; Guangyu Cheng, Qingjie Li, Lin Li, Qi Wang, Aidong Liu collected and analysed the data, while Guangyu Cheng wrote the manuscript.

\section{REFERENCES}

1. Li XX, Dong B, Lian YB, Li C. Effect of Tongxinluo Capsule Combined with Qiliqiangxin Capsule on Trop J Pharm Res, February 2019; 18(2): 374 
Vascular Endothelial Function and Heart Function in Patients with Chronic Congestive Heart Failure. Chin Arch Tradit Chin Med 2018; 3(7): 1753-1755.

2. Wang S, Hu YW, He FW. Effect of different doses of atorvastatin on vulnerable plaque, fibrous cap and lipid core angle of coronary arteries in patients with acute coronary syndrome. Zhejiang Med J 2017; 39 (17): 1465-1468.

3. Solymoss BC, Bourassa MG, Campeau L, Sniderman A, Marcil M, Lespérance J, Lévesque S, Varga S. Effect of increasing metabolic syndrome score on atherosclerotic risk profile and coronary artery disease angiographic severity. Am J Cardiol 2004; 93(2): 159-164.

4. Xu CF, Shang LWX, Liu T, Wang TF, Zhang C. Synthesis and Anti-myocardial Ischemia Activity of the Danshensu Ester Derivatives. Strait Pharm J 2018; 3(2): 13-18.

5. World Health Organization. Declaration of Helsinki. $\mathrm{Br}$ Med J 1996; 313(7070): 1448-1449.

6. Tang $Q Z$, Zhang $X P$, Chen $X Z$, Tang $Q$. Effect of salvianolate combined with atorvastatin on endothelial function and inflammatory factors in patients with acute coronary syndrome after percutaneous coronary intervention. Chin J Interv Cardiol 2015; 23(5): 282-285.

7. Wang J, He QY, Yao KW, Wang SH, Xing YW, Zhu FY. Study on evaluation criteria of combined efficacy of coronary heart disease with angina pectoris. $J$ Tradit Chin Med 2008; 49(9): 842-844.

8. Zhao SP. Clinical application of statins in the prevention and treatment of coronary heart disease. Chin J Cardiol 2003; 31(4): 316-318.
9. Hu CS, Wei YF, Hu DY. A stepped and synthetic strategy for clinical lipid-lowering therapy. Chin J New Drugs Clin Remedies 2006; 25(8): 625-626.

10. Shi CJ, Wang ZL, Zhao HC, Li Q, Chen GM, Zhang M, Shao $Z$. Therapeutic effect of sodium tanshinone IIA sulfonate combined with cinepazide maleate in the treatment of angina pectoris. Shandong Med J 2011; 51(17): 71-72.

11. Parrinello CM, Landay AL, Hodis HN, Gange SJ, Norris PJ, Young M, Anastos K, Tien PC, Xue XN, Lazar J, et al. Association of subclinical atherosclerosis with lipid levels amongst antiretroviral-treated and untreated HIV infected women in the Women's Interagency HIV study. Atheroscler 2012; 225(2): 876-877.

12. Qiu X, Miles A, Jiang $X$, Sun $X$, Yang $N$. Sulfotanshinone sodium inject ion for unstable angina pectoris: a systematic review of randomized controlled trials. Evid Based Complement Alternat Med 2014; 2012(3): 715790.

13. Zhao HJ, Zu DL, Jiang ZC. Clinical Observation of Shengmai Injection in Treating Coronary Heart Disease with Heart Failure. Strait Pharm J 2014; 23(5): 154-155.

14. Jeong JW, Lee B, Kim DH, Jeong HO, Moon KM, Kim MJ, Yokozawa T, Chung HY. Mechanism of Action of Magnesium Lithospermate $B$ against Aging and ObesityInduced ER Stress, Insulin Resistance, and Inflammsome Formation in the Liver. Mol 2018; 23(9): 2098. 